CLAWAR 2020: 23rd International Conference on

Climbing and Walking Robots and the Support

Technologies for Mobile Machines,

Moscow, Russian Federation, 24-26 August 2020

https://doi.org/10.13180/clawar.2020.24-26.08.52

\title{
Survey of Approaches for Emergency Landing Spot Detection with Unmanned Aerial Vehicles
}

\author{
G. Loureiro \\ Instituto Superior de Engenharia do Porto \\ Porto, Portugal \\ E-mail: 1170081@isep.ipp.pt \\ A. Dias and A. Martins \\ INESC TEC/ISEP \\ Porto, Portugal \\ E-mail: andre.dias@inesctec.pt, alfredo.martins@inesctec.pt
}

\begin{abstract}
For the past years, the interest in the use of Unmanned Aerial Vehicles (UAVs) has been increasing due to the multiple research topics provided by the field of aerial robotics. Conversely, vehicles are susceptible to failures or malfunctions. Consequently, one main emergent research topic is the detection of a safe landing spot in these emergency scenarios. Therefore, this paper exposes and details the multiple techniques that attempt to solve the problem of landing site detection. This paper aims to present the current literature with several sensors that can be used to solve the aforementioned problem. Finally, the paper presents our proposed approach with some preliminary results in simulation.
\end{abstract}

\section{Introduction}

In recent years, the research and use of UAVs, commonly known as drones, have been increasing substantially due to the large set of research topics, such as hardware development, human-system interaction, obstacle detection, and collision avoidance. ${ }^{1}$ These vehicles consist of an aircraft that can be remotely operated by a human operator or execute a mission autonomously. ${ }^{2}$ In the latter case, the degree of autonomy and the mission which they are capable to achieve depend on the sensors used and the level of on-board processing.

In terms of categorization, there are several ways in which UAVs can be classified: aerodynamics, landing, weight and range. ${ }^{3}$ Generally, the classification regarding their aerodynamics is commonly used. For instance, there are fixed-wing, flying-wings and flapping-wing vehicles in which their wings are the main factor to generate lift. On the other hand, there are other types such as helicopters, a quadcopter that uses multiple rotors to produce forceful thrust. Most of the current vehicles can be defined within those categories. ${ }^{4}$

Given the numerous UAVs with multiple sensors, motors and design configuration, they present applicability in several kinds of operations, such as search and rescue, ${ }^{5,6}$ delivery, ${ }^{7}$ surveillance, ${ }^{8}$ inspection and interaction with the environment. ${ }^{9,10}$ Considering the application scenarios, there are missions where the UAV must fly in civilian airspace, i.e., they must fly over populated areas. However, they are susceptible to external disturbance or electromechanical malfunction. As a matter of fact, there are different failures scenarios that could impact the operation thus leading to an emergency landing: software and hardware errors, energy limitation, loss of communication, Global Positioning System (GPS) failures and environment factors.

In this type of scenario, the UAVs must safely land in an area that minimizes damage to the robot and won't cause any injury to humans. Therefore, estimate a reliable landing spot is essential to safe operation. The problem of emergency landing can be branched in different steps: fault detection, detection, and evaluation of landing spot, path planning, and 


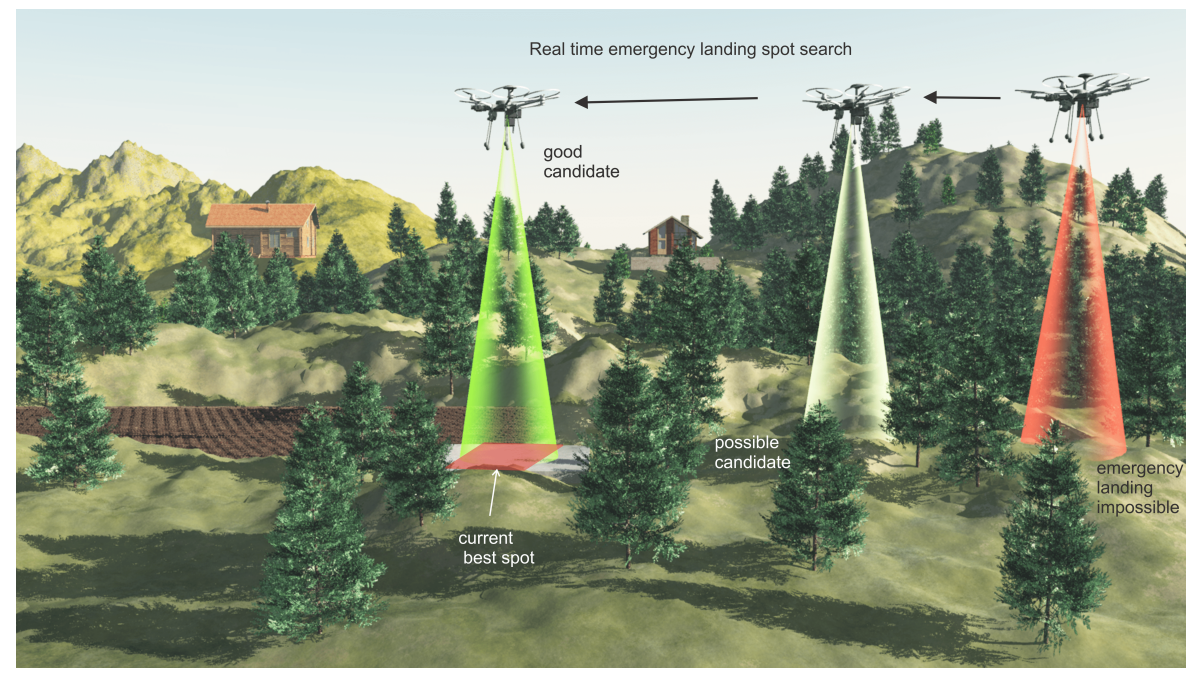

Fig. 1. Conceptual approach for Emergency landing spot detection with a UAV.

landing control. There are considerable works related to the different branches and the aim of this survey is to analyze the numerous methods regarding the detection and evaluation of the landing site. Despite the excellent survey from Kendoul, ${ }^{11}$ Kong, ${ }^{12}$ Gautam ${ }^{13}$ and Jin, ${ }^{14}$ the focus of their research were vision-based algorithm and control techniques for landing systems. For this reason, this work focus on different sensors based algorithms to the detection and assessment of the landing site in an emergency scenario.

Following this motivation, the paper outline is as follows. In section 2 we present the problem of landing spot detection. Then, we present the algorithms that use Light Detection And Ranging (LIDAR) as the main source of data in section 3. In section 4, vision-based methods are exposed. Section 5 presents works a set of sensors, such as Radio Detection And Ranging (RADAR), LiDAR and cameras to improve the reliability of the detected landing site. In Section 6 we present our algorithm and some simulation results. Finally, in section 7 we discuss the several methods presented and some conclusions.

\section{Problem Formulation}

The problem of landing site detection is subdivided into two parts: detection of a potential landing zone and assessment of the area. Considering that, the algorithm process sensor data to extract terrain information and applies a set of conditions to evaluate the suitability of the area. These conditions are typically restraints on the surface. The reliability of a safe landing site depends on two main factors, the distance of the aircraft to the landing site and the ground conditions. The ground conditions are all the factors that are relevant when the aircraft is in contact with the ground. Examples of the conditions that must be considered are:

- The slope of the plane;

- The roughness of the area;

- The size of the spot;

- Presence of obstacles;

- Distance to UAV Position.

Considering the multiples sensors that can be mounted in a UAV, there are multiples approaches to solve the described problem. For instance, LiDAR sensors provide a point cloud that can be processed to extract terrain features. On the other hand, cameras produce 
a 2-D representation of the world that a generally segmented to obtain useful information or to realize a $3-\mathrm{D}$ reconstruction.

\section{LiDAR Landing Detection Systems}

It is established in the literature that LiDAR sensors can provide accurate data about the environment and their scope of applicability ranges from terrestrial robots to aerial robots. Johnson et al. ${ }^{15}$ developed in 2002 a system that uses LiDAR data to detect obstacles and safely land spacecraft on Mars. Their system is based on geometric analysis of terrain characteristics. After generating a 2.5-D grid map to represent the terrain, it computes a Least Median Square estimation to fit a plane in a grid cell. The selection step is based on constructing a landing cost map given terrain roughness and incidence angles and choosing an optimum landing site.

Another algorithm that uses LiDAR data is presented by Whalley et al. ${ }^{16,17}$ and Takahashi et al. ${ }^{18}$ The author proposed a method that analyses a 3-D point cloud generated by a LiDAR to autonomously determine possible safe landing sites. The classification of the landing spots is realized by computing the plane of a set of points and applying geometrical constraints. These constraints are the slope of the computed plane that must be below a limit, the roughness and proximity of hazards and obstacles. The safe landing algorithm maps the 3-D point cloud data from each LiDAR scan on a grid. Next a square sliding window with the size of a landing area moves along the grid to compute the slope and roughness at each point. Then, the slope and roughness are analyzed to determine if the window is a feasible landing spot. After the entire grid is covered and the statistics are calculated, the optimal landing point is chosen. An optimum point has the minimum value of the sum of weighted roughness, slope, and distance.

A navigation system proposed by Chamberlain et al. ${ }^{19}$ allows a full-scale unmanned helicopter to fly through unmapped terrain, detect and perform a safe landing without human control or input. Their algorithm is described in Scherer et al. ${ }^{20-22}$ and consists of two evaluation steps based on geometrical features. The first step, labeled coarse evaluation, takes a 3-D point cloud registered in global coordinates as input to build a grid map in which each cell contains points and statistical information, such as mean, minimum and maximum height, number of points. Then, a first filtering step is applied where cells with standard deviation in $\mathrm{z}$ larger than a threshold are rejected. A plane fitting is applied to compute slope and roughness and find potential landing sites. In the final step, denoted fine evaluation, a 2-D Delaunay triangulation of the potential landing sites data is built and used to determine the intersections with the landing skids and computing the roll and pitch of the aircraft. Finally, the volume between the triangulation and a 3-D model of the helicopter is calculated. The value of the volume is used to predict bad contact and as another measure to classify the landing site. They realized experimental tests in urban and vegetated areas where their system was able to detect safe landing sites. The drawbacks of the algorithm are the detection of false positives in water and the rejection of potential landing sites in low vegetation.

The problem of considering low vegetation as roughness is addressed in Maturana and Scherer. $^{23}$ The authors proposed a 3-D Convolutional Neural Networks to detect landing zone for small UAVs. The algorithm incrementally creates a volumetric density map from the point cloud stream. The xy plane of the map is subdivided into non-overlapping tiles and for each tile, the terrain surface height is estimated. These sub-volumes are the input of the trained neural network. Finally, the output of the algorithm is the reliability rating of the analyzed sub-volume regarding the feasibility of land. With this approach, they were able to detect small obstacles presented in vegetation. 
The work of Lorenzo et al. ${ }^{24}$ in 2017 presents an algorithm for landing site detection in parallel on manycore systems. Their proposal is tested on several sets of data with different characteristics. First, the 3-D points of the point cloud are sorted to their $\mathrm{x}$ coordinates and then to their y coordinates. Second, a read-only octree is built to hasten the neighborhood search. ${ }^{25}$ The next step of the algorithm is to fit a plane in a neighborhood larger enough for the aircraft using Principal Component Analysis (PCA) ${ }^{26}$ The normals to all points are computed in parallel. Finally, a quality rate is assigned to the plane regarding the slope, roughness, skid landing requirements and presence of obstructions.

\section{Vision Landing Detection Systems}

In addition to the use of LiDAR, the vision system has been the most popular approach to detect and assess a landing spot. There are multiple strategies to land a UAV using visionbased algorithms. The focus of this survey is the works related to landing on an unknown or partially known site as it is the most likely to happen in an emergency. However, other strategies for known environments are landing on a marker, runway or a moving platform. ${ }^{12}$ The cameras have the advantage of being inexpensive and lighter compared to LiDARs. In Garcia-Pardo et al., ${ }^{27}$ a safe landing site is an area that is big enough for the helicopter to land and is clear of obstacles. Their algorithm assumes that contrast in an image is higher near the obstacles. Therefore, it searches circular areas in an image in which pixels have a level of contrast below a maximum value.

The work developed by Bosch et $a .^{28}$ presents a method to detect landing areas autonomously using monocular images. The algorithm applies a homography estimation process to select points that lie in a plane. Then, a dense correlation technique is used to distinguish planar surfaces from non-planar areas. Finally, the information is saved in a stochastic 2-D grid in which each cell has the probability of being planar. However, as the grid has the probability of the region being flat, it is not capable of being used for other functions, such as obstacle avoidance.

Another technique used to detect landing spots is machine vision. In Fitzgerald et al. ${ }^{29,30}$ and Mejias et al., ${ }^{31}$ a machine approach is used to locate safe landing areas for UAV forced landings given aerial imagery. The first step is a preliminary site selection in which a Canny Edge Detector and line-expansion algorithm is applied to generate two binary images. These images are fused and processed to generate a preliminary map where the safe and unsafe areas are labeled. The second step consists of classifying the type of surface and build a coarse slope map. Finally, an optimum landing map is built by fusing the previous maps using fuzzy linguistic as decision method. The work is later extended by Warren et al. ${ }^{32}$ in 2015. The 2-D points in the preliminary maps are projected into a $3-\mathrm{D}$ world model using the camera pose and intrinsic model, accounting for the terrain ruggedness. Then, a 3-D reconstruction using Structure-from-Motion is realized. Finally, the algorithm applies a 3-D surface analysis regarding size, local obstacles, terrain smoothness to select the landing spot.

Eendebak et al. ${ }^{33}$ proposed in 2013 an algorithm for emergency landing selection in realtime operations. The author aimed to detect landing zones considering moving objects. The authors used Background Estimation ${ }^{34}$ on stabilized video and then compared the difference between the current frame of the video and the background estimate to detect moving objects and structures. A binary image is generated indicating obstacles and a distance map to all detected obstacles is built. The maximum distance in the map is computed and chosen as the landing site.

In 2013, Shen et al. ${ }^{35}$ used cameras to acquire visual information and detect emergency landing sites autonomously. The method was validated in offline experiments. First, the ground in the image is identified by applying a hierarchical elastic horizon detection al- 
gorithm. After the horizon is detected, a Canny Edge Detector is applied to assess the roughness of the area. Furthermore, the terrain image is clustered in several clusters using the K-mean clustering method. Then, the clusters are processed and analyzed to detect the potential landing spot.

Forster et al. ${ }^{36}$ proposed an elevation map-based technique to landing spot detection for micro aerial vehicles using a monocular camera. The images from the camera and the vehicle's pose are combined to build a depth map. Moreover, an elevation map is generated and updated given the resulting maps. In the selection step, the authors considered a landing spot as a flat surface with a radius that depends on the size of the vehicle.

Recently, Hinzmann et al. ${ }^{37}$ developed a vision-based algorithm to detect landing spots in unknown environments at run-time. The proposed algorithm applies a segmentation technique to a camera image and classifies the resulting segmented regions as "grass" or "not grass". Then, 3-D reconstruction and consequently 2.5-D elevation map algorithms are realized given the potential landing sites from the segmentation step. The optimum landing site is chosen given terrain characteristics, such as slope and roughness, Finally, a distance map is built.

\section{Other approaches}

Besides the previous works presented, other approaches have been proposed in which multiple sensors are used to solve the problem of terrain assessment and safety. Serrano et al. ${ }^{38}$ proposed a combination of RADAR, LiDAR and camera and a probabilistic framework to increase the robustness of the selection of landing spots in landed space operation. A regression algorithm is applied to fit a plane using RADAR and LiDAR range data and determine slope and roughness. In addition, the authors used edge detection techniques to identify craters and rocks from camera imagery. Considering the terrain features, a Bayesian Networks $^{39}$ is used to assess ground safety. On their proposal, the potential landing quality is modeled using terrain, available fuel, and region interest.

A similar sensor set is used by Howard and Seraji. ${ }^{40}$ In this case, three hazard maps are built from RADAR, LiDAR, and camera and used to extract measurements and terrain features. Each map is associated with a confidence variable that designates the sensor certainty. Then, the different maps are aligned and combined into a single fused map using fuzzy logic that represents terrain safety.

\section{Proposed algorithm}

After realizing a thorough study on the existing techniques, we proposed an emergency landing spot detection system to determine potential landing zones during flight time. ${ }^{41}$ The algorithm (see Figure 2) uses LiDAR data and a geometrical approach to extract statistics and information of an unknown environment. Firstly, the system accumulates the point cloud until one of two conditions is met: the traveled distance is larger than a minimum value or the accumulated point cloud reached a maximum limit. The next step consists of down-sampling the cloud using a Voxel Grid Filter and then structuring it by using an octree. The potential landing spots are detected by fitting a plane using the PCA method in a spherical neighborhood of a point. The spots are rated and stored regarding their radius size, the standard deviation in $\mathrm{z}$, the slope, the distance to the UAV and the optimum spot is chosen. Finally, a re-evaluation is realized periodically to check if another spot is the new optimum landing spot in case of emergencies. The algorithm performance was evaluated in simulation using the Modular Open Robots Simulation Engine (MORSE). We developed an environment shown in Figure 3a to simulate a bad environment with high roughness and a few landing sites. The algorithm searched for spots with a maximum radius of 2 meters at 


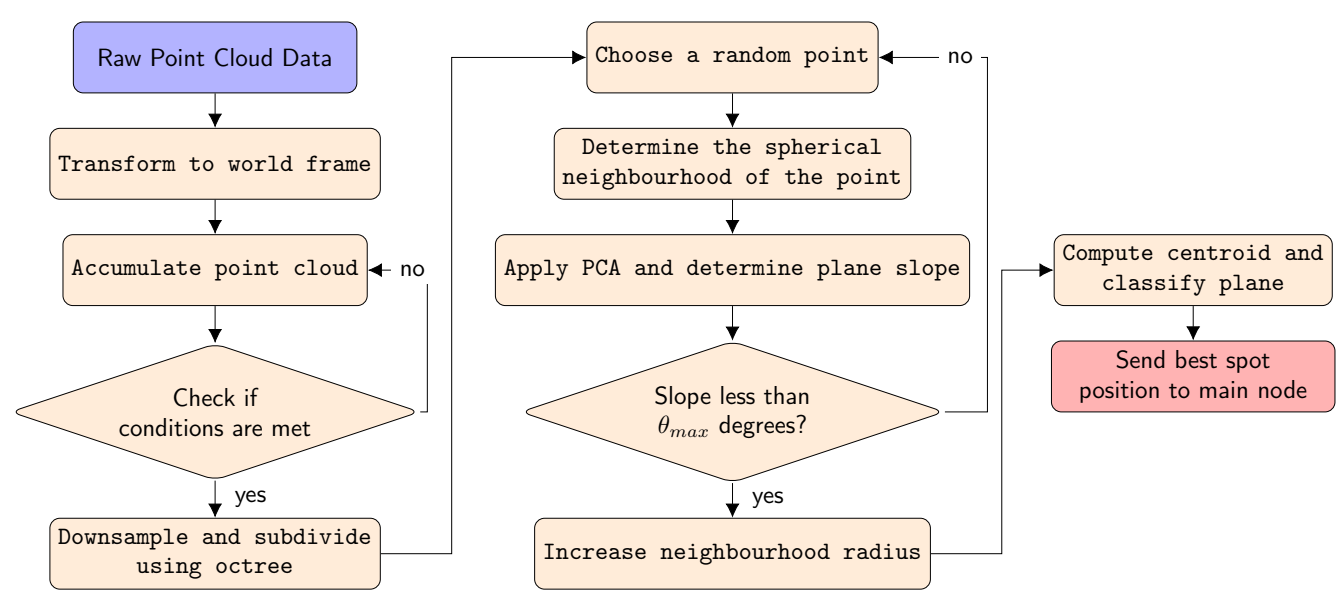

Fig. 2. Pipeline of the proposed algorithm.

the plane detection step. Despite not detecting all the feasible landing sites, the proposed system found different optimum spots as indicated by the black crosses in Figure 3b.

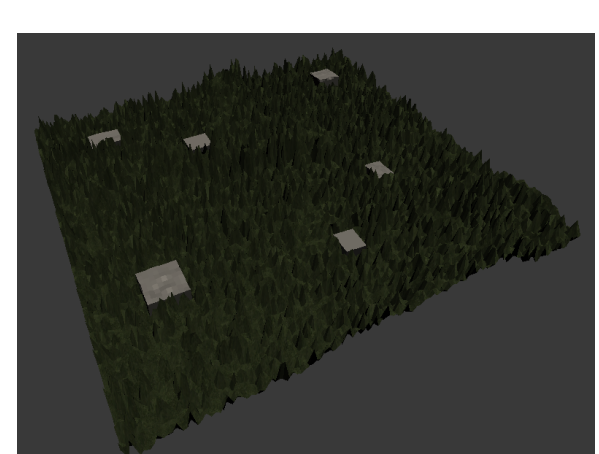

(a) Morse environment.

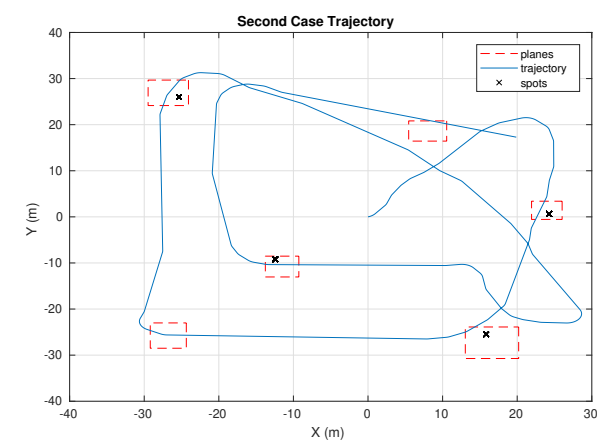

(b) Plot results for the simulation.

Fig. 3. Simulation environment in MORSE and results plot. ${ }^{41}$

\section{Discussion and Conclusions}

In this paper, we present the works related to the topic of detection, selection, and classification of safe landing sites. Some of the exposed approaches had already proposed solutions to overcome some limitations and drawbacks associated with the aforementioned problem. Considering the different sets of sensors, cameras are less expensive and lighter than other sensors. However, the applicability of a vision-based algorithm depends on visibility conditions. Conversely, LiDAR sensors are able to overcome this hindrance and present higher accuracy in more challenging environments. Nevertheless, LiDARs weight and processing power can be a restriction to some UAVs.

Specifically, the point cloud generated by LiDAR sensors must be spatially structured. Data structuring allows the storage and organization of information. In this context, this division leads to more efficient data access and process. Generally, the method chosen for structuring could cause loss of information like the 2.5-D grids and planes projected images. In order to overcome this hindrance, one proposal is to use an octree to structure the point 
cloud.

Regarding the detection of landing spots, the basis of the detection algorithm is to identify a plane on the surface. Several techniques for real-time implementation of those algorithms search for geometrical features, such as slope and roughness, to classify suitable landing sites in a point cloud. This approach does not present the need to train the algorithm when compared to approaches based on neural networks and machine vision. According to the exposed works, the detected spots must be classified on different levels considering the feasibility of landing. Hence, the final classification depends on terrain conditions, the presence of obstacles, distance to the UAV, remaining power and trajectory conditions. Considering our proposal, the algorithm is also able to detect landing spots and periodically sort them during the operation.

Finally, there are still some provoking topics: developing an efficient algorithm that runs in real-time given limited computational power; optimize the process of classifying landing spots considering the various factors that can influence the decision process; how to distinguish false positives, small obstacles.

\section{References}

1. K. A. Demir, H. Cicibas and N. Arica, Unmanned aerial vehicle domain: Areas of research., Defence Science Journal 65 (2015).

2. C. F. Liew, D. DeLatte, N. Takeishi and T. Yairi, Recent developments in aerial robotics: A survey and prototypes overview, arXiv preprint arXiv:1711.10085 (2017).

3. G. Singhal, B. Bansod and L. Mathew, Unmanned aerial vehicle classification, applications and challenges: A review (2018).

4. B. Vergouw, H. Nagel, G. Bondt and B. Custers, Drone technology: Types, payloads, applications, frequency spectrum issues and future developments, in The Future of Drone Use, (Springer, 2016) pp. 21-45.

5. P. Sousa, A. Ferreira, M. Moreira, T. Santos, A. Martins, A. Dias, J. Almeida and E. Silva, Isep/inesc tec aerial robotics team for search and rescue operations at the eurathlon challenge 2015, in 2016 International Conference on Autonomous Robot Systems and Competitions (ICARSC), 2016.

6. G. De Cubber, D. Doroftei, D. Serrano, K. Chintamani, R. Sabino and S. Ourevitch, The eu-icarus project: developing assistive robotic tools for search and rescue operations, in 2013 IEEE international symposium on safety, security, and rescue robotics (SSRR), 2013.

7. Amazon.com Inc., Amazon Prime Air https://www.amazon.com/primeair, Accessed on Feb. 11, 2020.

8. N. H. Motlagh, M. Bagaa and T. Taleb, Uav-based iot platform: A crowd surveillance use case, IEEE Communications Magazine 55, 128 (2017).

9. F. Azevedo, A. Dias, J. Almeida, A. Oliveira, A. Ferreira, T. Santos, A. Martins and E. Silva, Real-time lidar-based power lines detection for unmanned aerial vehicles, in 2019 IEEE International Conference on Autonomous Robot Systems and Competitions (ICARSC), 2019.

10. M. Aghaei, F. Grimaccia, C. A. Gonano and S. Leva, Innovative automated control system for pv fields inspection and remote control, IEEE Transactions on Industrial Electronics 62, 7287 (2015).

11. F. Kendoul, Survey of advances in guidance, navigation, and control of unmanned rotorcraft systems, Journal of Field Robotics 29, 315 (2012).

12. W. Kong, D. Zhou, D. Zhang and J. Zhang, Vision-based autonomous landing system for unmanned aerial vehicle: A survey, in 2014 international conference on multisensor fusion and information integration for intelligent systems (MFI), 2014.

13. A. Gautam, P. Sujit and S. Saripalli, A survey of autonomous landing techniques for uavs, in 2014 international conference on unmanned aircraft systems (ICUAS), 2014.

14. S. Jin, J. Zhang, L. Shen and T. Li, On-board vision autonomous landing techniques for quadrotor: A survey, in 2016 35th Chinese Control Conference (CCC), 2016.

15. A. E. Johnson, A. R. Klumpp, J. B. Collier and A. A. Wolf, Lidar-based hazard avoidance for safe landing on mars, Journal of guidance, control, and dynamics 25, 1091 (2002).

16. M. Whalley, M. Takahashi, P. Tsenkov, G. Schulein and C. Goerzen, Field-testing of a helicopter 
uav obstacle field navigation and landing system, in 65th Annual Forum of the American Helicopter Society, Grapevine, TX, 2009.

17. M. S. Whalley, M. D. Takahashi, J. W. Fletcher, E. Moralez III, L. C. R. Ott, L. M. G. Olmstead, J. C. Savage, C. L. Goerzen, G. J. Schulein, H. N. Burns et al., Autonomous black hawk in flight: Obstacle field navigation and landing-site selection on the rascal juh-60a, Journal of Field Robotics 31, 591 (2014).

18. M. D. Takahashi, A. Abershitz, R. Rubinets and M. S. Whalley, Evaluation of safe landing area determination algorithms for autonomous rotorcraft using site benchmarking, Journal of the American Helicopter Society 58, 1 (2013).

19. L. Chamberlain, S. Scherer and S. Singh, Self-aware helicopters: Full-scale automated landing and obstacle avoidance in unmapped environments

20. S. Scherer, L. Chamberlain and S. Singh, Autonomous landing at unprepared sites by a fullscale helicopter, Robotics and Autonomous Systems 60, 1545 (2012).

21. S. Scherer, L. Chamberlain and S. Singh, Online assessment of landing sites, in AIAA Infotech@ Aerospace 2010, 2010 p. 3358.

22. S. Scherer, Low-altitude operation of unmanned rotorcraft (2011).

23. D. Maturana and S. Scherer, 3d convolutional neural networks for landing zone detection from lidar, in 2015 IEEE international conference on robotics and automation (ICRA), 2015.

24. O. G. Lorenzo, J. Martínez, D. L. Vilariño, T. F. Pena, J. C. Cabaleiro and F. F. Rivera, Landing sites detection using lidar data on manycore systems, The Journal of Supercomputing 73, 557 (2017).

25. A. S. M. Mosa, B. Schön, M. Bertolotto and D. F. Laefer, Evaluating the benefits of octreebased indexing for lidar data, Photogrammetric Engineering \&6 Remote Sensing 78, 927 (2012).

26. K. Pearson, Liii. on lines and planes of closest fit to systems of points in space, The London, Edinburgh, and Dublin Philosophical Magazine and Journal of Science 2, 559 (1901).

27. P. J. Garcia-Pardo, G. S. Sukhatme and J. F. Montgomery, Towards vision-based safe landing for an autonomous helicopter, Robotics and Autonomous Systems 38, 19 (2002).

28. S. Bosch, S. Lacroix and F. Caballero, Autonomous detection of safe landing areas for an uav from monocular images, in 2006 IEEE/RSJ International Conference on Intelligent Robots and Systems, 2006.

29. D. Fitzgerald, R. Walker and D. Campbell, A vision based forced landing site selection system for an autonomous uav, in 2005 International Conference on Intelligent Sensors, Sensor Networks and Information Processing, 2005.

30. D. L. Fitzgerald, Landing site selection for uav forced landings using machine vision, $\mathrm{PhD}$ thesis, Queensland University of Technology2007.

31. L. Mejias, D. L. Fitzgerald, P. C. Eng and L. Xi, Forced landing technologies for unmanned aerial vehicles: towards safer operations, Aerial vehicles 1, 415 (2009).

32. M. Warren, L. Mejias, X. Yang, B. Arain, F. Gonzalez and B. Upcroft, Enabling aircraft emergency landings using active visual site detection, in Field and Service Robotics, 2015.

33. P. Eendebak, A. van Eekeren and R. den Hollander, Landing spot selection for uav emergency landing, in Unmanned Systems Technology XV, 2013.

34. M. Piccardi, Background subtraction techniques: a review, in 2004 IEEE International Conference on Systems, Man and Cybernetics (IEEE Cat. No. 04CH37583), 2004.

35. Y.-F. Shen, Z.-U. Rahman, D. Krusienski and J. Li, A vision-based automatic safe landing-site detection system, IEEE Transactions on Aerospace and Electronic Systems 49, 294 (2013).

36. C. Forster, M. Faessler, F. Fontana, M. Werlberger and D. Scaramuzza, Continuous on-board monocular-vision-based elevation mapping applied to autonomous landing of micro aerial vehicles, in 2015 IEEE International Conference on Robotics and Automation (ICRA), 2015.

37. T. Hinzmann, T. Stastny, C. Cadena, R. Siegwart and I. Gilitschenski, Free lsd: prior-free visual landing site detection for autonomous planes, IEEE Robotics and Automation Letters 3, 2545 (2018).

38. N. Serrano, A bayesian framework for landing site selection during autonomous spacecraft descent, in 2006 IEEE/RSJ International Conference on Intelligent Robots and Systems, 2006.

39. H. E. Kyburg, Probabilistic reasoning in intelligent systems: networks of plausible inference by judea pearl, The Journal of Philosophy 88, 434 (1991).

40. A. Howard and H. Seraji, Multi-sensor terrain classification for safe spacecraft landing, IEEE Transactions on Aerospace and Electronic Systems 40, 1122 (2004).

41. G. Loureiro, L. Soares, A. Dias and A. Martins, Emergency landing spot detection for unmanned aerial vehicle, in Iberian Robotics conference, 2019. 